\title{
Mitochondrial Apoptosis Reduces Mutagenesis Regardless Oxidative Stress
}

Marco Giorgio ${ }^{1 *}$, Antonella Ruggiero ${ }^{1,2}$ and Pier Giuseppe Pelicci ${ }^{1}$

${ }^{1}$ Department of Experimental Oncology, European Institute of Oncology, Via Adamello 16, 20139 Milan, Italy

${ }^{2}$ Department of Biological Regulation, Weizmann Institute of Science, 76100 Rehovot, Israel

\begin{abstract}
Upon oxidative stress DNA accumulates adducts and breaks that activate the genome damage response to repair, arrest and eventually suicide the damaged cell. Indeed, challenges with exogenous pro-oxidants increase mutations and reduce survival. Thus, the amounts of pro-oxidants generated by endogenous oxygen metabolism are thought to affect mutation frequency. However, oxidative stress induces cell death, clearing damaged cells.

The LacZ reporter system has been used previously to detect mutation rate in flies and mice. Recently, we have measured in vivo spontaneous mutation rate in mice with reduced mitochondrial ROS production and cell death rate, by crossing p66Shc or Cyclophilin D knockout mice, characterized by reduced intracellular concentration of reactive oxygen species and by impaired apoptosis, with a transgenic line harboring multiple copies of the LacZ mutation reporter gene.
\end{abstract}

Results indicated that the inhibition of endogenous oxidative stress and the following induced apoptosis increased genome rearrangements suggesting that specific genetic sets, in precise environments, determine somatic mutations rate.

Keywords: Mutagenesis; Reactive oxygen species; Apoptosis; Mitochondria

\section{Introduction}

DNA is vulnerable to an array of damaging agents of both, endogenous and environmental origin. Therefore, specialized DNA repair systems evolved to preserve nuclear DNA integrity. These systems are continuously required for the excision and replacement of damaged nucleotide residues. Indeed, if DNA damage is not promptly and faithfully repaired, DNA replication can fix the mutation. When levels of DNA damage are elevated, DNA repair systems become overwhelmed and mutations accumulate. The level of DNA repair activity declines with age [1] therefore the mutational burden of the cell increases as organisms become older [2]. Genomic instability is considered a major stochastic mechanism of ageing [3,4]. Moreover, accumulation of high mutation rates may lead to cell death, cancer, premature ageing and neurodegenerative diseases [5].

Somatic mutations, point mutations (transitions and transversions) and chromosome rearrangements, can be generated by an endogenous or an exogenous source of DNA damage. There is a broad variety of environmental factors that can cause the accumulation of mutations in living organisms, among them the chemicals formed during the combustion of sulfur-containing fossil and N-methyl-N-nitrosamine, present in tobacco smoke (Swann and Magee, 1968), mutagens present in food such as aflatoxins and UV radiation.

Reactive Oxygen Species (ROS) are highly reactive products of oxygen $\left(\mathrm{O}_{2}\right)$ reduction and include superoxide anion $\left(\mathrm{O}_{2}^{-} \cdot\right)^{\circ}$, hydrogen peroxide $\left(\mathrm{H}_{2} \mathrm{O}_{2}\right)$ and hydroxyl radical $\left(\mathrm{OH}^{-} \cdot\right)$. When high-energy radiations, such as $\mathrm{X}$ rays and gamma radiations, interact with water, ROS can be formed. They are also produced during normal cell metabolism by specific proteins. Life on earth is largely based on oxygen metabolism that is the driving force for the majority of reactions occurring in living organisms. As a general rule, reductive processes are used to store energy and oxidative processes to release it. Complete $\mathrm{O}_{2}$ reduction produces water, whereas its partial reduction produces ROS. The high reactivity of ROS is due to the presence of unpaired valence shell electrons. Hence, ROS can alter proteins and lipids as well as DNA. ROS cause modification of purine and pyrimidine bases, of the deoxyribose backbone, single and double strand breaks, as well as cross-links to other molecules. More than 20 different types of base damage have been identified. The most frequent damage to purines produces the formation of 8-oxo-dG (7,8-dihydro-8-oxoguanine), while for pyrimidines the formation of thymine glycol. Eight-oxo-dG [6] is generated when the $\mathrm{C} 8-\mathrm{OH}$ adduct radical, produced by the reaction between the highly reactive hydroxyl radical and guanine, loses an electron and a proton. The $\mathrm{C} 8-\mathrm{OH}$ adduct radical may also be reduced by uptake of an electron and a proton forming 7-hydro8-hydroxyguanine which is subsequently converted to 2,6-diamino-4hydroxy-5-formamidopyrimidine, a second major oxidation product of guanine. Mutations that may arise due to the formation of 8-oxoG involve GC $\rightarrow$ TA transversions [7].

Specialized repair proteins continuously scan the genome. When they encounter a lesion such as an AP site, a mismatched base or a structurally altered base, they start one of the DNA repair mechanisms that ultimately lead the restoration of the genetic information. DNA repair is carried out through several different pathways that process different types of DNA damage; these include BER, NER, homologous recombination, non-homologous end joining, and mismatch repair. When a lesion cannot be repaired, the only resource that allows cell survival is lesion bypass, operated by a process of trans-lesion synthesis

*Corresponding author: Marco Giorgio, Via Adamello 16, 20139, Milan, Italy, Tel: +39-02-94375040; Fax: +39-02-94375990; E-mail: marco.giorgio@ieo.eu

Received November 20, 2013; Accepted January 15, 2014; Published January 21, 2014

Citation: Giorgio M, Ruggiero A, Pelicci PG (2014) Mitochondrial Apoptosis Reduces Mutagenesis Regardless Oxidative Stress. J Carcinog Mutagen S3: 005. doi:10.4172/2157-2518.S3-005

Copyright: ( 2014 Giorgio M, et al. This is an open-access article distributed under the terms of the Creative Commons Attribution License, which permits unrestricted use, distribution, and reproduction in any medium, provided the original author and source are credited. 
in which a polymerase or replicative assembly encounters a non-coding or miscoding lesion, inserts an incorrect nucleotide opposite to the lesion and then continues elongation [8]. Eventually, DNA damage activates checkpoint regulators such as p53, SMC1, FANCD2, BRCA1 that promote cell cycle arrest and apoptosis. The impairment of any component of this machinery increases dramatically mutagenesis and promotes cancer. Indeed, clearance of damaged cells by apoptosis is considered the last barrier against the fixation of excessive deleterious mutations.

However, the role in vivo of ROS levels and apoptosis on mutagenesis has not been clearly defined.

\section{Endogenous ROS Functions}

ROS are highly reactive species that can exert mutagenic effects on both mitochondrial and nuclear DNA. Moreover, ROS can alter lipids and proteins. Hydroxyl radical produced by the reaction of $\mathrm{Fe}^{+}$and $\mathrm{H}_{2} \mathrm{O}_{2}$, for example, inactivates the Krebs cycle enzyme aconitase. In turn, it can contribute to further ROS generation which amplifies the damage. Oxidized proteins are recognized by proteases and degraded; new replacing proteins are synthesized de novo.

Specific enzymes such as superoxide dismutases (Mn-SOD), catalases (CAT), lactoperoxidases, glutathione peroxidases (Gpx) and peroxiredoxins (Prx) scavenge ROS levels to prevent the harmful consequences (oxidative stress) of their accumulation. The presence of small antioxidant molecules such as ascorbic acid, tocopherol, uric acid, and glutathione also helps to scavenge free radicals. Vitamin E, for example, can break the covalent links formed by ROS between fatty acid side chains in membrane lipids [9].

Superoxide dismutases represent the most important class of enzymes that defend cells from oxygen exposure. In mammals, three forms of superoxide dismutase have been described: SOD1 is located in the cytoplasm, SOD2 in the mitochondria and SOD3 in the extracellular space. They catalyze the dismutation of superoxide into $\mathrm{O}_{2}$ and $\mathrm{H}_{2} \mathrm{O}_{2}$. Mice deficient for SOD have a decreased life span and an elevated incidence of liver cancer [10] $\mathrm{H}_{2} \mathrm{O}_{2}$ is the most stable ROS; it can diffuse from mitochondria into the cytosol, where it is further metabolized by catalases, Gpx1 and PrxIII. Catalase is concentrated in peroxisomes located next to mitochondria and reacts with the $\mathrm{H}_{2} \mathrm{O}_{2}$ to catalyze the formation of $\mathrm{H}_{2} \mathrm{O}_{2}$ and $\mathrm{O}_{2}$. Mice lacking catalase exhibit a retarded rate in decomposing extracellular $\mathrm{H}_{2} \mathrm{O}_{2}$ with respect to wild type and a severe brain injury following physical trauma [11]. Notably, mice that overexpress mitochondrial catalase show an extended lifespan [12]. Glutathione peroxidase reduces $\mathrm{H}_{2} \mathrm{O}_{2}$ by transferring the energy of the reactive peroxides to a very short sulfur peptide called glutathione. Peroxiredoxins degrade $\mathrm{H}_{2} \mathrm{O}_{2}$ in the mitochondria, cytosol and nucleus compartments.

Regardless their damaging effect, ROS play important functions in the cell. They are essential for the defense of the organism against pathogens and they also play a major role in the regulation of intracellular signaling pathways [13].

Neutrophils kill engulfed pathogens by using myeloperoxidase, an enzyme that catalyzes the reaction of $\mathrm{H}_{2} \mathrm{O}_{2}$ with $\mathrm{Cl}^{-}$to produce the strongly antiseptic $\mathrm{ClO}^{-}$ion. In addition, neutrophils produce $\mathrm{ROS}$ by NADPH oxidase. This enzyme is also present in macrophages where localizes in the lysosome membrane and catalyzes the synthesis of superoxide anion to destroy the phagocitated host.

Moreover, NADPH oxidase has been found in cells, such as fibroblasts, endothelial cells, osteoclasts and chondrocytes [14] where they have no role in host defense. The rate of ROS generation by $\mathrm{NADPH}$ oxidase in these cells is lower compared with neutrophils. This suggests, together with the different localization of the enzyme, that ROS, in these cells, are involved in signal transduction pathways [15]. Indeed, ROS are implicated in cell growth and differentiation, both activating and inhibiting apoptosis, and, at higher concentrations, inducing necrosis [16].

DNA microarray analysis have shown that $\mathrm{H}_{2} \mathrm{O}_{2}$ regulates the expression of almost 100 genes [15] and the activation of transcription factors such as NF-kB [17] and AP-1 [18], Myb and Ets [19] . Moreover, several enzymes involved in cell signaling are regulated by $\mathrm{H}_{2} \mathrm{O}_{2}$; for example, $\mathrm{H}_{2} \mathrm{O}_{2}$ produced in response to stimulation with Epidermal Growth Factor can cause reversible inactivation of Protein Tyrosine Phosphatase 1B [20] and PTEN [21] by oxidizing its catalytic site cysteine.

$\mathrm{H}_{2} \mathrm{O}_{2}$ does also regulate the opening of the mitochondrial permeability transition pore (MPTP), which plays a key role in the intrinsic apoptotic pathway. Indeed, opening of the MPTP alters mitochondrial membrane permeability and allows solutes to diffuse from mitochondria to cytoplasm, in particular apoptogenic factors, such as cytochrome $c$ that once released in the cytosol triggers the apoptotic cascade.

\section{P66Shc Regulates Mitochondrial ROS Production and Apoptosis}

P66Shc is one of the 3 isoforms encoded by the human and mouse ShcA locus. ShcA was identified in 1992 by low-stringency hybridization to human cDNA libraries, using a $\mathrm{SH} 2$-coding sequence as a probe [22]. It is located on chromosome 1q21. The originally isolated shcA transcript displayed two in-frame ATGs and was shown to encode two ubiquitously expressed polypeptides: p52Shc and p46Shc.

Members of the Shc family are characterized by the PTB-CH1SH2 modularity where: $\mathrm{CH} 1$ (collagen homologues 1) is a glycine/ proline rich region, containing 2 major phosphorylation sites: Y239240 and Y317; SH2 (Src homologous type 2) is the C-terminal domain involved in protein-protein interaction and mediates the formation of multi-protein signaling complexes; PTB (phosphotyrosine binding domain) is also able to bind non-phosphorylated Tyr-containing peptides. P66Shc contains an additional amino-terminal proline rich region, named $\mathrm{CH} 2$.

Isoforms p46Shc and p52Shc serve as phosphotyrosine adaptor molecules in various receptor-mediated signaling pathways. Once phosphorylated, they recruit the GRB2/SOS complex to the plasma membrane [23] and subsequently activate the mitogenic-activated protein kinase (MAPK) cascade. P52Shc and p46Shc function as initiators of the Ras signaling cascade in various non-neuronal systems [24]. P66Shc is a target of receptor tyrosine kinases [25] and it is able to bind the Grb2/SOS complex. However, p66Shc is not involved in Ras signaling regulation and its overexpression has a negative effect on the RAS-MAPK-fos pathway in response to EGF. On the contrary p66Shc converts intracellular oxidative signals into apoptosis [26]

A fraction of p66Shc has been observed within the mitochondrial inter-membrane space (IMS) .However Import mechanism of p66Shc into mitochondrial IMS has not been clearly identified. During apoptosis levels of p66Shc in mitochondria increase. Some stress kinases such as Jnk-1 and Pkc-B phosphorylate p66Shc in Ser36, and Pin-1 induces its prolil-isomerization [27]; these post-translational modifications 
are involved in p66Shc translocation within mitochondria. In basal conditions, mitochondrial p66Shc associates to a high molecular weight complex of about $670 \mathrm{kDa}$ and to the mitochondrial chaperon mtHsp70 [28] and components of TIMTOM import complex [29]. Notably, treatment of cells with pro-apoptotic stimuli such as UVC or $\mathrm{H}_{2} \mathrm{O}_{2}$ induces the dissociation of these complexes and the release of monomeric p66Shc), which then reacts with cytochrome $c$ [28] sequestering electrons from the ETC to generate $\mathrm{H}_{2} \mathrm{O}_{2}[30,31]$.

Deletion of p66Shc gene in mice results in increased resistance to oxidative stress [26] and reduced body weight (due to reduced fat accumulation) [32]. Interestingly these mice have an approximately $30 \%$ extension of life span. Notably, p66Shc deletion in mice improves resistance to hyperglycemic damage in a diabetic model of nephropathy as well as to cardiovascular disease, due the reduction of apoptosis and cell loss $[33,34]$.

P66Shc knock out (p66Shc-/-) fibroblasts are resistant to apoptosis induced by many signals, relating to the increased $\mathrm{H}_{2} \mathrm{O}_{2}$-sensitivity conferred by over expression of p66Shc [26].Being the production of energy mainly associated with glycolysis instead of ETC [35], p66Shc-/cells show a reduced mitochondrial metabolism.

P66Shc is not only involved in ROS generation, it regulates the expression of ROS scavenging enzymes through inhibition of FOXO transcription factors [36]. Indeed, in adipocytes, p66Shc is a downstream target of the insulin signaling pathway [31]. Activation of p66Shc leads to the activation of AKT by the direct effect of p66Shcgenerated $\mathrm{H}_{2} \mathrm{O}_{2}$ on PTEN. The effect of p66Shc is more marked on FOXO, a critical transcription factor downstream of AKT [31,37].

In animal tissues, the extent of oxidative damage correlates with the levels of expression of p66Shc. Indeed, oxidative damage is higher in the lung, spleen, liver, and skin where p66Shc is expressed at high levels, whereas no significant oxidative damage is found in brain where p66Shc is expressed at low levels [38]. Notably, the amount of 8-oxo-dG in nuclear and mitochondrial DNA was found to be lower in p66Shc-/mice.

\section{Mutation Assays In Vivo: Biological Systems}

Several approaches can be used to study mutation accumulation in vivo. In the past, it was only possible to detect chromosome rearrangements via cytogenetic analysis on proliferating cells, such as lymphocytes. Later, also point mutations could be detected in such cells, using selectable marker genes. The most popular was the X-chromosomal gene for hypoxanthine-guanine phosphoribosyltransferase (HPRT) locus test [39].

This enzyme converts purines (hypoxanthine, guanine, or 6-mercaptopurine) to the corresponding nucleoside monophosphates. Accumulation of these molecules in cells has cytotoxic effects. The assay is based on the fact that if mutations occur in the Hprt locus the function of the HPRT enzyme is compromised and cells are able to survive treatment with purine analogues.

This method was largely used to study accumulation of mutations with age in lymphocytes from humans and mice. The problem related to this assay is that it poorly reflects the in vivo system. The analysis is limited to active proliferating cells whereas the majority of adult cells only rarely undergo cell division.

With the advent of transgenic mouse technology, a completely different approach became possible. The use of reporter vectors integrated in mouse chromosomes allows studying mutations accumulation in every rodent organ. Reporter vectors are plasmids or phage packaging containing prokaryotic genes that can be easily recovered in host bacteria (mutated in the gene used as reporter) [40].

Vectors appear to be transcriptionally inactive as indicated by heavy methylation of CpG sites and absence of mRNA [41,42]; therefore mutations of the transgene are neutral.

The spontaneous mutation frequency in transgenic portions of the genome appears to be somehow higher than in endogenous genome, however some comparisons indicate that transgenes and endogenous genes have essentially the same reaction upon treatment with mutagens [43]. Endogenous genes are likely to differ substantially respect to mutation susceptibility and spectrum, due to variations like size, genomic location and function in a given organ or tissue. They are transcriptionally active and thus are subject to transcription-coupled DNA repair (TCR). It is unlikely that adducts in the transgene would be subject to TCR, they would rather be processed in a much slower timeframe via GGR [44].

Unrepaired DNA lesions that persist through DNA replication are clearly of most concern in tissues with a rapid turnover. In post-mitotic organs, mutations may be fixed by DNA repair process: for some types of repair, such as repair of double strand breaks, this may be significant.

Several types of shuttle vector-containing transgenic mouse models are now available. The first was described by Gossen and Vijg [45] and uses bacteriophage $\lambda$. The transgenic model was originally constructed by microinjection of the bacteriophage $\lambda$ gt10-lac $Z$ shuttle vector into fertilized CD2 mouse oocytes. lac $Z$ is part of lac operon, required for the transport and metabolism of lactose in bacteria. lac $Z$ encodes for the enzyme $\beta$-galactosidase that catalyzes the hydrolysis of lactose into glucose and galactose. Rescue of the vectors is performed by exposing total genomic DNA, isolated from different organs or tissues, to an $E$. coli in vitro packaging extract. In order to avoid host restriction, both the packaging extract and the plating cells need to be derived from a completely host restriction negative E. coli strain, such as E. coli $C$ [46]. The phages obtained are plated on a $\triangle$ lacZ E. coli strain for the selection of mutant and non-mutant lacZ phages.

Another transgenic model, based on the lac operon, was developed by Kohler to measure DNA mutation rate [47].The mutational target is lacI instead of lacZ. LacI protein negatively regulates lacZ expression; it acts by binding the operator sequence upstream of the lacZ gene when lactose is absent. Consequently, in this system, infection of lacZ $+E$. coli host cells allows the selection of phages contain a mutant or nonmutant lacI gene.

Both the lacZ and the lacI bacteriophage lambda systems have now been extensively used for studying mutation accumulation in vivo for genetic toxicological analysis. There are other systems based on bacteriophage $\lambda$ : the cII selection [48]. Glazer et al. developed transgenic mice equipped with a $\lambda$ supF shuttle vector [49] and Malling and Burkhart generated the phiX174 am3 cs70 transgenic mouse system [50].

In addition to $\lambda$ based models, mouse carrying plasmid vectors have been generated. The main differences between these models consist in the size of the shuttle vector and in how it is rescued. Unlike the phage based system, in which very high molecular weight genomic DNA must be isolated in order to obtain intact vectors with high efficiency, plasmids can be isolated from genomic DNA with a very high efficiency since multi-copy concatemers are integrated in mouse genome.

With the $\lambda$ system, it is impossible to detect deletions and insertions 
larger than $5 \mathrm{~kb}$ because in vitro packaging requires $\lambda$ vector with a minimum size of $42 \mathrm{~kb}$ and a maximum size of $52 \mathrm{~kb}$. The plasmid model, on the contrary, allows to identify deletions arising within the concat 3 mer as well as deletions extending from a lacZ target gene into flanking chromosomal sequences that can be recovered, detected and characterized [51].

Several lacZ transgenic lines were derived by microinjection and bred to homozygosity. In our experiments, we used C57BL/6J line 30 that harbors 21 copies of pUR288 tandemly integrated on chromosome 11. Line 60 harbors a total of about 20 copies of the plasmid integrated in a head-to-tail fashion at two chromosomal sites: chromosome 3 and chromosome 4. It was demonstrated that both lines give comparable results in mutagenesis studies [52]. Other plasmid transgenic mouse models were generated: rpsL transgenic mouse [53] (and 6-TG selection assay [54].

LacZ-plasmid mice have been extensively characterized, and mutation accumulation over time in several organs has been described. At young age, mutation spectra rates are similar, whereas over time small intestine, characterized by a very high cell proliferative activity, undergoes the largest age-related increase in mutation rate. In liver and heart, mutant frequencies increase with age from birth to 34 months; in brain, testes and spleen an increase is observed only between birth and 4-6 months [2]. Also the types of mutations accumulated vary from organ to organ: for example in small intestine the age-related increase is entirely due of point mutations, while in liver both point mutations and genome rearrangements occur.

\section{Mitochondrial Ros and Apoptosis Effect on Lacz Mutation Rate: The Lesson from P66shc}

We measured the accumulation of mutations in p66Shc-/- mice using the LacZ reporter transgenic mouse model. Double heterozygous (LacZ+/- -p66Shc+/-) first generation mice (F1) were crossed in order to obtain LacZ+/+-p66Shc-/- animals. The genotyping strategy used to assess the presence of the LacZ transgene does not to discriminate between $\mathrm{LacZ}+/+$ and LacZ+/- animals. Therefore, F2 mice that were p66Shc-/- were crossed with a WT C57/Bl6 mouse and genotype of the progeny was used to determine the genotype of the F2 mouse. Indeed, all mice of the progeny are $\mathrm{LacZ}+/$ - when a $\mathrm{LacZ}+/+$ animal is crossed with a WT mouse. F2 LacZ+/- mice, on the contrary, generate both, LacZ-/- and LacZ+/- progeny. F2 LacZ+/+ p66Shc-/- mice were crossed to establish the colony.

LacZ Plasmids were integrated head to tail in the mouse genome. To determine mutation frequency, lac $Z$ transgenes were rescued from the genomic DNA by excision with HindIII, followed by purification using magnetic beads, pre-coated with LacI repressor protein. Plasmids were then ligated and transferred to E. coli $C$ ( $\left.\triangle l a c Z, G a l E^{-}\right)$using electro-transformation. 1:1000 of transformants was plated in medium with X-gal to determine the total number of plasmids rescued. The remainder was plated in the presence of high concentrations of the lactose analogue p-gal that only allowed cells harboring a mutant lac $Z$ gene to grow. Mutants were characterized using restriction analysis. Results revealed [55] that size change mutations rather than no change mutations accumulated particularly in MEFs and small intestine from p66Shc-/- mice.

ROS induce DNA adducts and breaks that activate the genome damage response to induce repair, cell cycle arrest and eventually clear the damaged cell.

Indeed, studies on p66Shc-/- mice as other transgenic animals have

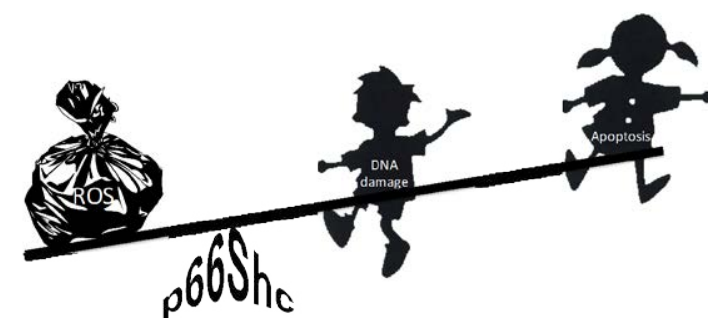

Figure 1: The characteristic p66Shc enzymatic machinery generates ROS that trigger preferentially apoptosis rather than damage DNA

shown that intracellular levels of ROS negatively correlate with stress resistance and life span [26]

We have found that the overall LacZ mutation frequency was unaffected by the deletion of p66Shc indicating that the amount of ROS generated by p66Shc, although relevant to trigger mitochondrial apoptosis, are not genotoxic. Indeed, p66Shc-/- mice showed normal tumor incidence.

However, the deletion of p66Shc changes the type of mutations that are accumulated over time and upon stress, as size change mutations were significantly higher in LacZ p66Shc-/- than in LacZ background, both in the small intestine from old and young irradiated mice and stressed MEFs.

Interestingly, LacZ and p66Shc-/- MEFs treated with $\mathrm{H}_{2} \mathrm{O}_{2}$ show similar frequency of size change mutations. Different size change frequency of MEFs treated with $\mathrm{H}_{2} \mathrm{O}_{2}$ suggests that susceptibility to die of the p66Shc-/- cells is independent to DNA damage. In agreement with this hypothesis we have observed that the deletion of cyclophilin $\mathrm{D}$ (CypD), that induces apoptosis through the opening of mitochondrial permeability transition pore with the same mechanism of p66Shc, increases size change mutations in the small intestine of old mice [55].

P66Shc and CypD are expressed in the liver and the fact that the small intestine but not the liver showed differences in the mutation rate compared to the $\mathrm{WT}$, suggests that tissues with high turnover particularly feel the effect of p66Shc and CypD, thus supporting the hypothesis that impaired apoptosis plays a role in mutation rate.

Finally, p66Shc deletion protects from stress and degenerative diseases but may affect the integrity of some tissues allowing the accumulation of particularly mutated cells (Figure 1).

To what extend these cells guarantee tissue function is questionable. Recently, we have found that p66Shc deletion is counter-selected when mice are maintained in harsh conditions (open field under cold and competition for food) that mimic wild, indicating that p66Shc is essential for fitness under stressing natural conditions but redundant in protected environment [56]. So, p66Shc-/- mice can survive to accumulate a peculiar spectrum of mutations only in laboratory conditions.

In conclusion, the particular spectrum of LacZ mutations in p66Shc-/- underlines the importance of cell death rather than the redox induced mutagenesis and suggests that specific genetic sets, in precise environments, determine somatic mutation rate.

\section{References}

1. Chen D, Cao G, Hastings T, Feng Y, Pei W, et al. (2002) Age-dependent decline of DNA repair activity for oxidative lesions in rat brain mitochondria. $J$ Neurochem 81: 1273-1284. 
Citation: Giorgio M, Ruggiero A, Pelicci PG (2014) Mitochondrial Apoptosis Reduces Mutagenesis Regardless Oxidative Stress. J Carcinog Mutagen S3: 005. doi:10.4172/2157-2518.S3-005

2. Vijg J, Dollé ME (2002) Large genome rearrangements as a primary cause of aging. Mech Ageing Dev 123: 907-915.

3. 3.Blagosklonny MV, Campisi J, Sinclair DA, Bartke A, Blasco MA, et al. (2010) Impact papers on aging in 2009. Aging (Albany NY) 2: 111-121.

4. Dollé ME, Snyder WK, Gossen JA, Lohman PH, Vijg J (2000) Distinct spectra of somatic mutations accumulated with age in mouse heart and small intestine. Proc Natl Acad Sci U S A 97: 8403-8408.

5. Boerrigter ME, Vijg J (1993) Studies on DNA repair defects in degenerative brain disease. Age Ageing 22: S44-52.

6. Valavanidis A, Vlachogianni T, Fiotakis C (2009) 8-hydroxy-2' -deoxyguanosine (8-OHdG): A critical biomarker of oxidative stress and carcinogenesis. J Environ Sci Health C Environ Carcinog Ecotoxicol Rev 27: 120-139.

7. Cheng KC, Cahill DS, Kasai H, Nishimura S, Loeb LA (1992) 8-Hydroxyguanine, an abundant form of oxidative DNA damage, causes G----T and A----C substitutions. J Biol Chem 267: 166-172.

8. Walker GC (1995) SOS-regulated proteins in translesion DNA synthesis and mutagenesis. Trends Biochem Sci 20: 416-420.

9. Haklar G, Sirikçi O, Ozer NK, Yalçin AS (1998) Measurement of reactive oxygen species by chemiluminescence in diet-induced atherosclerosis: protective roles of vitamin $\mathrm{E}$ and probucol on different radical species. Int J Clin Lab Res 28 : 122-126.

10. Busuttil RA, Rubio M, Dollé ME, Campisi J, Vijg J (2006) Mutant frequencies and spectra depend on growth state and passage number in cells cultured from transgenic lacZ-plasmid reporter mice. DNA Repair (Amst) 5: 52-60.

11. Ho YS, Xiong Y, Ma W, Spector A, Ho DS (2004) Mice lacking catalase develop normally but show differential sensitivity to oxidant tissue injury. J Biol Chem 279: 32804-32812

12. Schriner SE, Linford NJ, Martin GM, Treuting P, Ogburn CE, et al. (2005) Extension of murine life span by overexpression of catalase targeted to mitochondria. Science 308: 1909-1911.

13. Dröge W (2002) Free radicals in the physiological control of cell function Physiol Rev 82: 47-95.

14. Jones RD, Hancock JT, Morice AH (2000) NADPH oxidase: a universal oxygen sensor? Free Radic Biol Med 29: 416-424.

15. Hancock JT, Desikan R, Neill SJ (2001) Role of reactive oxygen species in cell signalling pathways. Biochem Soc Trans 29: 345-350.

16. Gamaley IA, Klyubin IV (1999) Roles of reactive oxygen species: signaling and regulation of cellular functions. Int Rev Cytol 188: 203-255.

17. Schreck R, Rieber P, Baeuerle PA (1991) Reactive oxygen intermediates as apparently widely used messengers in the activation of the NF-kappa B transcription factor and HIV-1. EMBO J 10: 2247-2258.

18. Rao GN, Glasgow WC, Eling TE, Runge MS (1996) Role of hydroperoxyeicosatetraenoic acids in oxidative stress-induced activating protein 1 (AP-1) activity. J Biol Chem 271: 27760-27764.

19. Myrset AH, Bostad A, Jamin N, Lirsac PN, Toma F, et al. (1993) DNA and redox state induced conformational changes in the DNA-binding domain of the Myb oncoprotein. EMBO J 12: 4625-4633.

20. Lee SR, Kwon KS, Kim SR, Rhee SG (1998) Reversible inactivation of proteintyrosine phosphatase 1B in A431 cells stimulated with epidermal growth factor. J Biol Chem 273: 15366-15372.

21. Lee SR, Yang KS, Kwon J, Lee C, Jeong W, et al. (2002) Reversible inactivation of the tumor suppressor PTEN by H2O2. J Biol Chem 277: 20336-20342.

22. Pelicci G, Lanfrancone L, Grignani F, McGlade J, Cavallo F, et al. (1992) A novel transforming protein ( $\mathrm{SHC}$ ) with an $\mathrm{SH} 2$ domain is implicated in mitogenic signal transduction. Cell 70: 93-104

23. Ravichandran KS (2001) Signaling via Shc family adapter proteins. Oncogene 20: $6322-6330$

24. Migliaccio E, Mele S, Salcini AE, Pelicci G, Lai KM, et al. (1997) Opposite effects of the p52shc/p46shc and p66shc splicing isoforms on the EGF receptor-MAP kinase-fos signalling pathway. EMBO J 16: 706-716.

25. Okada S, Kao AW, Ceresa BP, Blaikie P, Margolis B, et al. (1997) The 66-kDa Shc isoform is a negative regulator of the epidermal growth factor-stimulated mitogen-activated protein kinase pathway. J Biol Chem 272: 28042-28049.
26. Migliaccio E, Giorgio M, Mele S, Pelicci G, Reboldi P, Pandolfi, et al. (1999) The p66shc adaptor protein controls oxidative stress response and life span in mammals. Nature 402: 309-313.

27. Pinton P, Rimessi A, Marchi S, Orsini F, Migliaccio E, et al. (2007) Protein kinase $C$ beta and prolyl isomerase 1 regulate mitochondrial effects of the lifespan determinant p66Shc. Science 315: 659-663.

28. Orsini F, Migliaccio E, Moroni M, Contursi C, Raker VA, et al. (2004) The life span determinant p66Shc localizes to mitochondria where it associates with mitochondrial heat shock protein 70 and regulates trans-membrane potential. $J$ Biol Chem 279: 25689-25695

29. Cosentino F, Francia P, Camici GG, Pelicci PG, Lüscher TF, et al. (2008) Fina common molecular pathways of aging and cardiovascular disease: role of the p66Shc protein. Arterioscler Thromb Vasc Biol 28: 622-628.

30. Giorgio M, Migliaccio E, Orsini F, Paolucci D, Moroni M, et al. (2005). Electron transfer between cytochrome $c$ and p66Shc generates reactive oxygen species that trigger mitochondrial apoptosis. Cell 122: 221-233.

31. Trinei M, Migliaccio E, Bernardi P, Paolucci F, Pelicci P, et al. (2013) p66Shc mitochondria, and the generation of reactive oxygen species. Methods Enzymo 528: 99-110.

32. Berniakovich I, Trinei M, Stendardo M, Migliaccio E, Minucci S, et al. (2008) p66Shc-generated oxidative signal promotes fat accumulation. J Biol Chem 283: 34283-34293.

33. Camici GG, Schiavoni M, Francia P, Bachschmid M, Martin-Padura I, et al. (2007). Genetic deletion of p66(Shc) adaptor protein prevents hyperglycemiainduced endothelial dysfunction and oxidative stress. Proc Natl Acad Sci U S A 104: 5217-5222.

34. Carpi A, Menabò R, Kaludercic N, Pelicci P, Di Lisa F, et al. (2009) The cardioprotective effects elicited by p66(Shc) ablation demonstrate the crucia role of mitochondrial ROS formation in ischemia/reperfusion injury. Biochim Biophys Acta 1787: 774-780.

35. Nemoto S, Combs CA, French S, Ahn BH, Fergusson MM, et al. (2006) The mammalian longevity-associated gene product p66shc regulates mitochondria metabolism. J Biol Chem 281: 10555-10560.

36. Nemoto S, Finkel T (2002) Redox regulation of forkhead proteins through p66shc-dependent signaling pathway. Science 295: 2450-2452.

37. Trinei M, Berniakovich I, Beltrami E, Migliaccio E, Fassina A, et al. (2009) P66Shc signals to age. Aging (Albany NY) 1: 503-510.

38. Trinei M, Giorgio M, Cicalese A, Barozzi S, Ventura A, et al. (2002). A p53 p66Shc signalling pathway controls intracellular redox status, levels of oxidation-damaged DNA and oxidative stress-induced apoptosis. Oncogene 21: 3872-3878.

39. Albertini RJ (2001) HPRT mutations in humans: biomarkers for mechanistic studies. Mutat Res 489: 1-16.

40. Vijg J, van Steeg H (1998) Transgenic assays for mutations and cancer: current status and future perspectives. Mutat Res 400: 337-354.

41. Ikehata $H$, Takatsu M, Saito Y, Ono T (2000) Distribution of spontaneous CpGassociated G:C --> A:T mutations in the lacZ gene of Muta mice: effects of CpG methylation, the sequence context of $\mathrm{CpG}$ sites, and severity of mutations on the activity of the lacZ gene product. Environ Mol Mutagen 36: 301-311.

42. Monroe JJ, Manjanatha MG, Skopek TR (2001) Extent of CpG methylation is not proportional to the in vivo spontaneous mutation frequency at transgenic loci in Big Blue rodents. Mutat Res 476: 1-11.

43. Tao KS, Urlando C, Heddle JA (1993) Comparison of somatic mutation in a transgenic versus host locus. Proc Natl Acad Sci U S A 90: 10681-10685.

44. Lambert IB, Singer TM, Boucher SE, Douglas GR (2005) Detailed review of transgenic rodent mutation assays. Mutat Res 590: 1-280.

45. Gossen JA, de Leeuw WJ, Tan CH, Zwarthoff EC, Berends F, et al. (1989) Efficient rescue of integrated shuttle vectors from transgenic mice: a model for studying mutations in vivo. Proc Natl Acad Sci U S A 86: 7971-7975.

46. Gossen JA, Vijg J (1988) E. coli C: a convenient host strain for rescue of highly methylated DNA. Nucleic Acids Res 16: 9343.

47. Kohler SW, Provost GS, Fieck A, Kretz PL, Bullock WO, et al. (1991) Spectra of spontaneous and mutagen-induced mutations in the lacl gene in transgenic mice. Proc Natl Acad Sci U S A 88: 7958-7962. 
Citation: Giorgio M, Ruggiero A, Pelicci PG (2014) Mitochondrial Apoptosis Reduces Mutagenesis Regardless Oxidative Stress. J Carcinog Mutagen S3: 005. doi:10.4172/2157-2518.S3-005

48. Leach EG, Gunther EJ, Yeasky TM, Gibson LH, Yang-Feng TL, et al. (1996) Frequent spontaneous deletions at a shuttle vector locus in transgenic mice. Mutagenesis 11: 49-56.

49. Malling HV, Burkhart JG (1989) Use of phi X174 as a shuttle vector for the study of in vivo mammalian mutagenesis. Mutat Res 212: 11-21.

50. Vijg J, Dollé ME, Martus HJ, Boerrigter ME (1997) Transgenic mouse models for studying mutations in vivo: applications in aging research. Mech Ageing Dev 98: 189-202.

51. Vijg J, Dolle ME, Martus HJ, Boerrigter ME (1997a) Transgenic mouse models for studying mutations in vivo: applications in aging research. Mech Ageing Dev 99: 257-271
52. Gondo Y, Shioyama Y, Nakao K, Katsuki M (1996) A novel positive detection system of in vivo mutations in rpsL (strA) transgenic mice. Mutat Res 360: 1-14

53. Nohmi TKM, Suzuki H, Matsui M, Yamada M, Watanabe M, Suzuki M, et al. (1996) A new transgenic mouse mutagenesis test system using Spi- and 6-thioguanine selections. Environ Mol Mutagen 28, 465-470.

54. Beltrami E, Ruggiero A, Busuttil R, Migliaccio E, Pelicci PG, et al. (2013) Deletion of p66Shc in mice increases the frequency of size-change mutations in the lacZ transgene. Aging Cell 12: 177-183.

55. Giorgio M, Berry A, Berniakovich I, Poletaeva I, Trinei M, et al. (2012) The p66Shc knocked out mice are short lived under natural condition. Aging Cell 11: $162-168$

This article was originally published in a special issue, DNA damage/ repair: Mutagenesis : Carcinogenesis handled by Editor(s). Dr. Lubomir Manolov Stoilov, University: Institute of Genetics, Bulgaria; Dr. Kandace Jo Williams, University of Toledo College of Medicine, USA; Dr. Mu Wang, Indiana University School of Medicine, USA 